\title{
What makes recognition without awareness appear to be elusive? Strategic factors that influence the accuracy of guesses
}

\author{
Joel L. Voss ${ }^{1,3}$ and Ken A. Paller ${ }^{2}$ \\ ${ }^{1}$ Beckman Institute for Advanced Science and Technology, University of Illinois Urbana-Champaign, Urbana, Illinois 61801, USA; \\ ${ }^{2}$ Interdepartmental Neuroscience Program and Department of Psychology, Northwestern University, Evanston, Illinois 60208, USA
}

\begin{abstract}
Various factors could conceivably promote the accuracy of guesses during a recognition test. Two that we identified in previous studies are forced-choice testing format and high perceptual similarity between the repeat target and novel foil. In restricted circumstances, the relative perceptual fluency of the target can be compared with that of the foil and used as a reliable cue to guide accurate responses that occur without explicit retrieval-a phenomenon we referred to as "implicit recognition." In this issue, Jeneson and colleagues report a failure to replicate accurate guesses and also a tendency on the part of subjects to hazard guesses infrequently, even though testing circumstances were very similar to those that we used. To resolve this discrepancy, we developed a simple manipulation to encourage either guessing or confident responding. Encouraging guessing increased both the prevalence of guesses and the accuracy of guesses in a recognition test, relative to when confident responding was encouraged. When guessing was encouraged, guesses were highly accurate (as in our previous demonstrations of implicit recognition), whereas when confident responding was encouraged, guesses were at chance levels (as in Jeneson and colleagues' data). In light of a substantial literature showing high accuracy despite low confidence in certain circumstances, we infer that both the prevalence and accuracy of guessing can be influenced by whether subjects adopt guessing-friendly strategies. Our findings thus help to further characterize conditions likely to promote implicit recognition based on perceptual fluency.
\end{abstract}

In several prior experiments, we reported findings indicative of recognition without awareness (Voss et al. 2008; Voss and Paller 2009). The experiments involved recognition tests for colorful and complex geometric shapes (kaleidoscope images). Subjects attempted to discriminate repeat stimuli (targets) from novel stimuli (foils). In some of our experiments, subjects made recognition responses and also rated the quality of their recognition experience or their confidence in their decision. For example, recognition often occurred with awareness of memory retrieval and with some level of confidence. On the other hand, correct recognition of a target sometimes occurred with no discernable awareness of memory retrieval or confidence; essentially, subjects felt that their response was merely a guess-and yet they were correct.

Of course, the reason that a guess might be correct in a recognition test might have nothing at all to do with the subject having retrieved relevant information; the response might be merely a "lucky guess." Our results, however, provided evidence that processes of implicit memory were operative in producing at least a subset of the correct guesses. In recognition tests using a forced-choice format, targets and foils shared a high degree of perceptual similarity and were displayed side-by-side, and we found that, for guess responses, the repeat stimulus was correctly selected remarkably often. With no stored information (and given that the target occurred equally often on the left side and the right side, and that targets and foils were counterbalanced across subjects), the repeat stimulus should be selected correctly $50 \%$ of the time in the long run. In our original report, we found that $82 \%$ of the guess responses were correct, which was more accurate

${ }^{3}$ Corresponding author.

E-mail joelvoss@illinois.edu; fax (847) 491-7859.

Article is online at http://www.learnmem.org/cgi/doi/10.1101//m.1896010. than responses when trials with high- or low-confidence responses were pooled together (56\%; data combined for all study conditions) (Experiment 2 of Voss et al. 2008). We referred to this phenomenon as recognition without awareness or implicit recognition. For the present discussion, we will use the latter term.

Indeed, our results provided several additional reasons for linking implicit memory with this phenomenon of implicit recognition. In one experiment, each trial was classified as either (1) a recognition experience in which subjects recollected episodic information from their initial experience with the target; (2) recognition with familiarity for the target, but no other recall of prior information concerning the target; or (3) a guess with no confidence in the accuracy of the response (Voss and Paller 2009). We found that guesses were approximately as accurate as recollection responses (73\% vs. 79\%, respectively, averaged across encoding conditions), and that guesses were more accurate than decisions based on familiarity (59\%, averaged across encoding conditions).

Another feature of these experiments was that we contrasted two types of learning conditions. In one condition, to-be-remembered stimuli were viewed while subjects simultaneously performed a verbal working memory task. This task required that the subject listen to a spoken digit on each trial and respond according to whether the digit on the prior trial was odd or even (i.e., a one-back task). In the other condition, there were no spoken digits, and attention could be allocated fully to viewing the to-be-remembered stimuli. In several different experiments, recognition accuracy was higher with divided-attention study than with full-attention study. Although this is a highly unusual outcome for recognition performance, it was clear that divided attention during the study led to relatively less confidence during the recognition test, such that guessing was more prevalent, and these guess responses were highly accurate. 
Notably, these two key results-highly accurate guessing, and a recognition advantage for divided over full attention at study-were not obtained when recognition was tested with a yes-no format (targets and highly similar foils randomly intermixed and shown one stimulus at a time), or when a forced-choice test was prepared such that each target was paired with a random foil rather than a highly similar foil (Voss et al. 2008). On the basis of these findings, as well as additional results from electrophysiological recordings (described below in the Discussion section), we argued that subjects were able to weigh the relative perceptual fluency of the target and the foil only for forced-choice tests with high target/foil similarity, and then they could use this fluency cue to guide accurate selection of the target (Voss and Paller 2009).

We aim to develop a line of reasoning to clarify why implicit recognition might tend to operate preferentially in certain circumstances, such as when the relative perceptual fluency of targets versus foils is likely to serve as a useful cue, and when the ability to remember specific stimulus details does not provide a useful cue (as is the case when these details are largely shared between the target and the foil). In many situations, however, perceptual fluency may not be a good basis for making recognition judgments. Often, accurate recognition reflects conceptual elaboration about the meaning of an event, and the conceptual features are typically remembered more robustly than the set of stimulus features perceived during the course of the event. Thus, implicit recognition may be less likely to guide a response in a recognition test in the presence of confident memory for the target. Dividing attention during encoding resulted in lower confidence during the recognition test, and this may have been one factor that promoted reliance on signals of relative perceptual fluency. Of course, there may be other factors that also promote or inhibit this type of strategy in a recognition test.

\section{The role of guessing in implicit recognition}

Jeneson et al. (2010) reported three studies in which they failed to replicate our findings of implicit recognition. They did not find accurate guess responses, despite reproducing many aspects of our experimental design. ${ }^{4}$ Indeed, the various conditions included in our demonstrations of implicit recognition were reproduced in many respects (i.e., the same stimulus set, stimulus presentation times, etc.), such that trivial explanations for their failure to replicate can be dismissed. They interpreted this failure to replicate in light of the conventional wisdom that confidence and accuracy are highly correlated in recognition memory tests, and therefore that implicit recognition is an unprecedented and counterintuitive finding. They emphasized the divergence between implicit recognition and other memory phenomena, and concluded that implicit recognition is not robust, but rather is elusive; a reader of this study might wonder whether it is nonexistent.

\footnotetext{
${ }^{4}$ Seven subjects were tested in their first experiment, and $38 \%$ of the stimuli were the same as we used in our experiments (the rest were of similar format). The same auditory one-back task was used to divide attention during the study for half of the study test blocks, and recognition was tested using only a forced-choice format. Their second experiment included 24 subjects with the same stimuli and general procedures as their first experiment, but with the addition of a subset of the instructions for describing highconfidence, low-confidence, and guess responses that we used in our experiments. Their third experiment included 24 subjects using the same stimuli and presentation parameters as in Voss et al. (2008), including testing recognition with a yes-no format in half of the blocks, and with a forced-choice format in the other half.
}

On the contrary, the literature review below provides precedence for expecting confidence to sometimes clash with accuracy. Furthermore, we propose that useful explanatory power can be gained from the juxtaposition of our findings with those of Jeneson et al. (2010), and by placing these recognition studies within the context of a broader literature. This exercise provides an opportunity to better understand the circumstances necessary for demonstrating this exceptional phenomenon of implicit recognition.

Accordingly, we review below a set of reports of phenomena that bear some similarity to our reports of implicit recognition. Although these are far from the only examples of remarkably accurate guessing and efficacious "unconscious" cognition (e.g., Schooler et al. 1993; Hassin et al. 2005; Dijksterhuis et al. 2006, 2009), this brief literature review provides clues concerning how the Jeneson et al. (2010) experiments may have inadvertently been conducted differently from our own studies (Voss et al. 2008; Voss and Paller 2009). Following this review, we report new findings from an experiment that directly tested the effects of the putative methodological differences on implicit recognition. The end product, then, is an explanation that can reconcile these divergent results by appealing to strategic factors that may play an essential role in implicit recognition.

\section{Discrimination without awareness-an implicit recognition analog}

To better understand the conditions in which implicit recognition can be demonstrated, we first consider demonstrations of perceptual discrimination without awareness. Peirce and Jastrow (1884) provided evidence for highly accurate guess responses in perceptual discrimination judgments. Subjects (Peirce and Jastrow themselves) attempted to discriminate the difference between two physical weights of nearly the same mass. Despite claiming to guess (that is, reporting no confidence in the discrimination), accuracy in several separate experiments using similar methods averaged $\sim 70 \%$ (where chance was $50 \%$ ), and was as high as $90 \%$ in one experiment involving hundreds of trials. These findings were subsequently replicated in group studies of naïve subjects (Fullerton and Cattell 1892).

Later experiments by Sidis (1898) and Stroh et al. (1908) examined the accuracy of guesses in visual discrimination. In these studies, untrained subjects attempted to identify a single letter printed on paper and held at viewing distances so far away that the letter appeared as a gray dot without discernable structure. Subjects were given a pool of letters from which to choose, and therefore the task was to discriminate the visual information from a set of mental representations of letters. Despite claims from subjects such as "I might as well close my eyes and guess," accuracy was always significantly above chance. This result was found repeatedly in multiple experiments, and was over four times chance-level accuracy in some experiments.

In a summary of research on these sorts of behaviors that occur without awareness of the relevant ability, Adams (1957) indicated that accuracy was significantly above chance in every published experiment in which perceptual discriminations were made when subjects claimed to be guessing (Fig. 1). Summarizing results from experiments similar to those described above, this investigator concluded: "... the only type of behavior without awareness which can be easily reproduced on the basis of published reports is the classical type, in which $S$ knows what he is supposed to be discriminating, but does not know that he is discriminating, because of the absence of the usual sensory 
experiences to which he is accustomed under the given type of stimulation" (Adams 1957).

In what ways are the circumstances needed to accurately solve perceptual discriminations when guessing similar to those needed to demonstrate implicit recognition? In both situations, subjects must discriminate between two or more choices with high perceptual overlap. Highly accurate guess responses in perception fail in circumstances that do not involve discrimination between two or more choices, such as when there is no accessible preexisting representation or other stimulus against which the current discriminandum can be compared (Adams 1957). Similarly, we found highly accurate guesses during forced-choice tests, but did not find evidence for accurate guesses during yesno format testing when targets and foils were presented individually (Voss et al. 2008). Likewise, targets and foils must be of high perceptual similarity for accurate guesses in perceptual discrimination. As suggested in the quote above, this creates a situation in which the large perceptual discrepancies that normally exist between two discriminanda are unavailable, and therefore guessing occurs. Likewise, our use of geometric stimuli comprised of difficult-to-verbalize features along with attentional diversions that further limit deliberate and/or semantic encoding is intended to effectively eliminate the conceptual information on which individuals normally base episodic memory judgments, thereby promoting guessing.

To summarize, numerous studies show that perceptual discriminations can be made with high accuracy but little or no awareness of that accuracy. As such, these results mirror the dissociations observed in patients with blindsight (see further discussion of blindsight below). Interestingly, circumstances necessary for these highly accurate guesses are very similar to those we used to investigate implicit recognition. Yet, one important difference between perceptual discriminations without awareness and implicit recognition concerns the nature of the signal that drives the highly accurate guess responses. Unlike in perceptual discrimination, implicit recognition involves discriminating

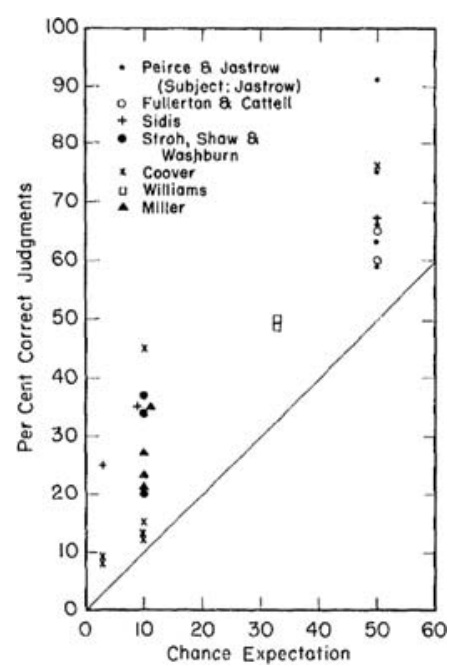

Figure 1. Surprisingly accurate perceptual discriminations without awareness. The average accuracy achieved for responses that subjects categorized as "blind guesses" is shown for each experiment reviewed by Adams (1957), and the accuracy that would be expected by chance is indicated by the solid line. The included experiments concerned perceptually demanding forced-choice format discriminations, and all were group experiments except for the report by Peirce and Jastrow (1884). Also see Table 2 of Adams (1957). Figure reproduced from Adams (1957) with permission from APA (c) 1957. based on memory signals (which we ascribe to perceptual fluency, as described below). We therefore now consider evidence for the role of memory signals in forced-choice discrimination in circumstances in which accuracy and awareness of that accuracy (confidence) are inversely related.

\section{Confidence/accuracy dissociations in forced-choice recognition}

In typical recognition-testing circumstances, confidence and accuracy are highly correlated. That is, when subjects report high confidence in recognition decisions, the accuracy of those decisions is higher than when subjects report lower confidence (Kelley and Wixted 2001; Yonelinas 2001). Likewise, manipulations that influence confidence also influence accuracy (e.g., both are enhanced by semantic processing during the study phase [Craik and Lockhart 1972], both are reduced by diverting attention during the study phase [Mulligan 1998], etc.). This is consistent with commonsense notions about recognition memory, in that introspection generally suggests that high certainty happens for a reason.

However, this strong relationship between confidence and accuracy has been identified almost entirely in recognition memory circumstances in which there is ample dissimilarity between targets and foils used in the test (i.e., study a set of images of common objects and later test discrimination of repeat objects from a set of other objects with different semantic and perceptual features such as name, natural category, size, shape, and so on). That is, in typical memory experiments, targets and foils do not overlap on a large set of perceptual and conceptual dimensions. Furthermore, dissimilarities between targets and foils are likely enhanced by the common practice of using memoranda that are highly familiar, such as words and nameable pictures. This reliance on nameable stimuli might also encourage a reliance during recognition testing on memory signals that can be introspectively verbalized (e.g., recollection and familiarity), rather than on implicit signals that cannot be verbalized (as in verbalovershadowing effects) (e.g., Schooler et al. 1993).

In our experiments with complex kaleidoscopic shapes, in contrast, we identified high accuracy without confidence (implicit recognition) using targets and corresponding foils with high perceptual similarity and without preexisting conceptual/ semantic representations. What, then, is the relationship between accuracy and confidence in forced-choice recognition circumstances in which target/foil similarity is high?

Tulving (1981) was the first to describe an inverse relationship between confidence and accuracy when recognition memory was tested using targets and foils with high similarity. High target/ foil similarity was achieved by using pictures of the same natural scenes taken from two slightly different vantage points. Effects of high target/foil similarity on recognition confidence and accuracy were compared with standard circumstances in which target/foil similarity was low (with pictures of different scenes). Across two studies using different ranges of target/foil similarity, accuracy increased as the perceptual similarity of targets and their foils increased, whereas confidence decreased. That is, confidence was inversely related to accuracy, an "inversion" of the normal relationship (high confidence $=$ high accuracy, low confidence $=$ low accuracy) observed when targets and foils are not highly similar.

Several studies have since replicated inversions between confidence and accuracy, all using targets and foils with high perceptual similarity. Dobbins et al. (1998) used pictures of scenes and a design similar to Tulving's (Tulving 1981), and found the same inversion effect. Heathcote et al. $(2009,2010)$ used 
unfamiliar human faces as stimuli and varied target/foil perceptual similarity, again showing the confidence/accuracy inversion effect. It is important to note that subjects in studies showing confidence/accuracy inversions cannot, in general, have valid awareness of accuracy. This is because confidence/accuracy inversion effects are not compatible with the notion that subjects were truly aware of their accuracy, as awareness of accuracy would produce positively correlated confidence and accuracy. It is unclear whether the same basic mechanisms were responsible for both the highly accurate guessing that we observed and the confidence/accuracy inversion effects in these previous studies, but we consider this a reasonable possibility given the highly similar circumstances across these studies (e.g., forced-choice recognition testing, high target/foil perceptual similarity).

Importantly, testing was done with a forced-choice format in all studies finding confidence/accuracy inversions with perceptually similar targets and foils (Fig. 2). As noted by Tulving (1981), this inversion has not been found in yes-no format tests. In the context of this highly consistent pattern of inverse relationships between confidence and accuracy with high target/foil perceptual similarity, the findings of Jeneson et al. (2010) stand out in that they found the standard rather than the inverted confidence/ accuracy relationship despite using highly similar targets and foils. What factors might have contributed to the absence of confidence/accuracy inversion effects in the results of Jeneson et al. (2010)?

\section{Hazarding a guess}

As noted above, highly accurate guesses are the key finding that we label as implicit recognition, and highly accurate guesses could be more generally responsible for the confidence/accuracy inversions reported by Tulving (1981) and others. Furthermore, guessing may be the key feature of the divergence between our findings and those of Jeneson et al. (2010). That is, Jeneson and colleagues reported guess responses that were no better than chance, whereas guess responses in our experiments were significantly above chance. Jeneson and colleagues also reported a different relationship between diverting attention during the study and the prevalence of guess responses than we found in our experiments. In our experiments (Voss et al. 2008, Experiment 2; Voss and Paller 2009), diverting attention during encoding led to a guess rate 2.6 times greater than that observed with full-attention encoding, whereas Jeneson et al. (2010) reported a divided-attention guess rate only 1.4 times greater than that observed with full-attention encoding (averaged across all experiments in each case). Indeed, the prevalence of guess responses does not appear to be significantly greater for divided compared with full attention in any of the Jeneson et al. (2010) experiments (based on the mean and variance information provided in their Table 1). Their subjects apparently did not resort to guessing more frequently when deprived of the superior encoding that occurs with full attention (e.g., verbal/semantic analyses that typically facilitate subsequent explicit memory). In contrast, subjects in our experiments did guess significantly more often when encoding had been disrupted with divided attention. This divergence in guessing is particularly striking in light of the fact that so many experiment parameters (stimuli, presentation timing, etc.) were matched between the Jeneson et al. (2010) studies and our previous studies. Could the propensity to hazard a guess when explicit memory was weak underlie the differences in guess accuracy between our experiments and those of Jeneson and colleagues? If this were the case, then we would conclude that the type of strategy adopted by the subject during memory testing has a critical influence on implicit recognition.

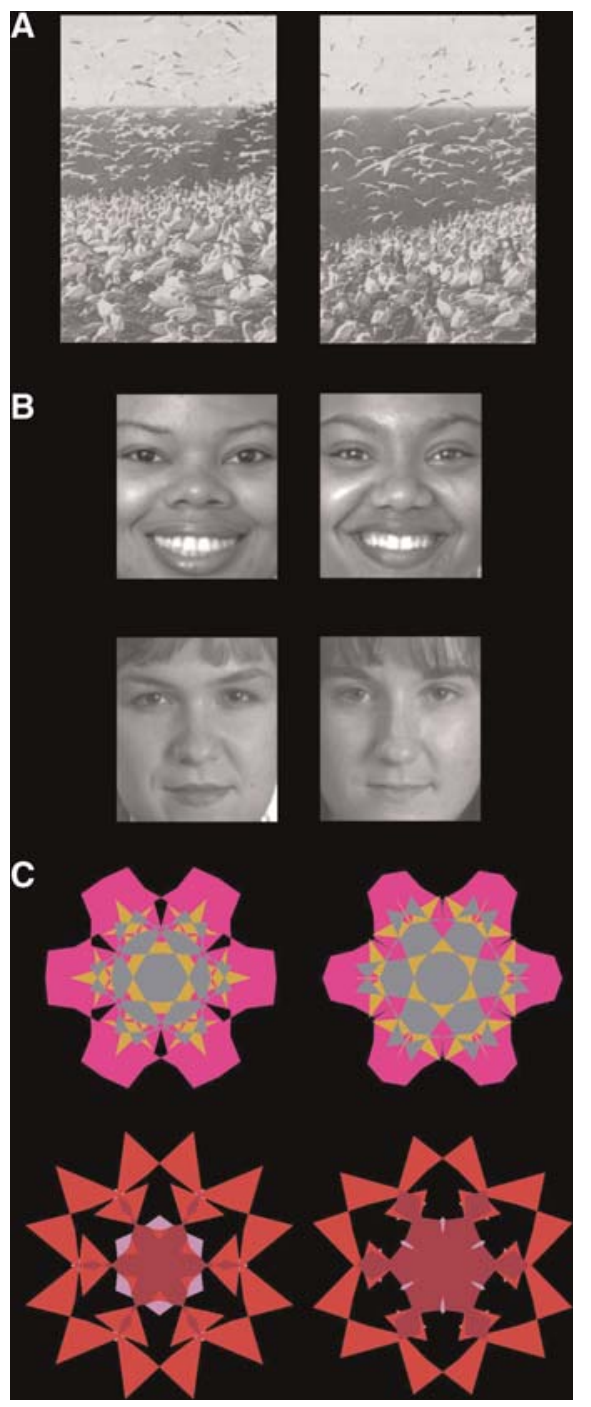

Figure 2. Target/foil pairs of high perceptual similarity in experiments that have identified inverse relationships between confidence and accuracy with forced-choice format recognition tests. Representative stimuli are shown in $A$ from the experiments by Tulving (1981) and Dobbins et al. (1998), in B from the experiments by Heathcote et al. (2009, 2010), and in C from experiments on implicit recognition (the current experiment and Voss et al. 2008; Voss and Paller 2009; Jeneson et al. 2010). Panel $A$ reproduced from Tulving (1981) with permission from Elsevier (c) 1981. Panel $B$ reproduced from Heathcote et al. (2009) with permission from The Psychonomic Society, Inc. (c) 2009.

The experiments by Sidis (1898) and Stroh et al. (1908) regarding highly accurate perceptual discriminations provide a clear illustration for why the propensity to guess is important (as do other studies reviewed by Adams 1957). They report that subjects objected to making extremely difficult perceptual differences (e.g., "I might as well close my eyes and guess"). If subjects were unwilling to hazard a guess, or if they had actually closed their eyes, then accuracy would not have been as surprisingly high as was reported. Moreover, if subjects were instructed to perform the task in such a way that accurate, confident responses were emphasized, they might have failed to use whatever minimal perceptual information was available when making the discriminations, thus also producing low guess response rates and low accuracy. In our experiments, we attempted to make subjects feel at ease in making guesses, emphasizing repeatedly during 
Table 1. Response rate and proportion correct for remember, know, and guess responses when guessing or confidence was encouraged

\begin{tabular}{lccccc}
\hline & \multicolumn{2}{c}{ Guessing encouraged } & & \multicolumn{2}{c}{ Confidence encouraged } \\
\cline { 2 - 3 } & Rate & $\mathbf{P}($ cor $)$ & & Rate & $\mathbf{P}($ cor $)$ \\
\hline Remember & $0.27(0.04)$ & $0.76(0.06)$ & & $0.36(0.03)$ & $0.80(0.04)$ \\
Know & $0.48(0.03)$ & $0.62(0.03)$ & & $0.52(0.03)$ & $0.60(0.03)$ \\
Guess & $0.26(0.04)$ & $0.78(0.06)$ & & $0.12(0.02)$ & $0.43(0.07)$ \\
\hline
\end{tabular}

the experiment that guessing was an acceptable response, and that they should simply remain attentive and go with their "gut feelings" if they were not immediately confident about their recognition response.

Yet another telling example of why the propensity to guess is important comes from the literature on blindsight (e.g., Weiskranz 1990). If a cortically blind patient has no awareness of information in the visual modality within a scotoma, there is subjectively no basis for producing the correct answer for a visual discrimination, and no reason to make any decision about visual stimulation within the scotoma at all. Yet, if the patient is willing to guess, as in a two-alternative forced-choice testing format, the guess may reveal residual visual information.

In this sense, we postulate that there are two different types of guess. An ordinary guess is one based on the complete absence of relevant information, in which case a guess is just a guess. Other times, a guess can be based on relevant information that is present in brain networks, but that is not available for conscious introspection. So a blindsight patient may have a choice of either relying on a search for the same type of information that arises from visual stimulation outside of the scotoma, or relying on implicit visual information. More generally, in order to make a guess informed by implicit knowledge, an individual needs to be willing to allow this specific information to dictate the response. This distinction is akin to the difference between analytic and nonanalytic processing that has been proposed in the memory literature (Whittlesea and Price 2001), whereby encouraging more part-based versus more holistic strategies can influence even simple perceptual priming phenomena (Willems and Van der Linden 2009).

We hypothesize that the way in which subjects were led to approach the task in the Jeneson et al. (2010) experiments may have inadvertently emphasized retrieval strategies that discouraged guessing. In our new experiment, we tested this hypothesis by replicating the conditions that achieved the strongest evidence for implicit recognition previously (kaleidoscope stimuli, forced-choice testing, encoding with divided attention, high target/foil perceptual similarity), but with a new manipulation. In $50 \%$ of the experimental trial blocks, subjects were encouraged to guess (as in our original studies), whereas, in the other $50 \%$, confident responding was encouraged.

\section{Results}

Encouraging guessing versus encouraging confident responding significantly influenced subjective reports of confidence (i.e., retrieval awareness) that were registered following recognition responses. The guess rate was approximately twice as high in blocks when guessing was encouraged compared with blocks when confident responding was encouraged [26\% of all responses vs. $12 \%$ of all responses, respectively, $t_{(23)}=4.5, P=0.0002$ ]. Importantly, this was not the only effect of the manipulation. Accuracy of guess responses was also significantly influenced, consistent with the reasoning above, reaching a mean accuracy of
$77.7 \%$ correct when guessing was encouraged and 43\% correct when confident responding was encouraged $\left[t_{(23)}=4.0, P=\right.$ 0.001]. Guess responses were significantly more accurate than expected by chance (50\%) when guessing was encouraged $\left[t_{(23)}=4.9, P<0.0001\right]$. On the other hand, guess accuracy did not differ significantly from chance when confident responding was encouraged $\left[t_{(23)}=1.0, P=0.32\right]$.

In order to ensure that differences between guess accuracy for blocks in which guessing was encouraged versus blocks in which confident responding was encouraged were not an artifact of low trial counts, we conducted an additional analysis using a subset of the data for which trial counts were never very low. Six subjects registered $<10 \%$ of all responses as guesses for either the encourage-guessing condition (one subject) or the encourageconfidence condition (five subjects). After removing data from these subjects, guesses were still more prevalent for blocks in which guessing was encouraged versus blocks in which confident responding was encouraged [ $26 \%$ vs. $16 \%$, respectively, $t_{(17)}=3.9$, $P=0.001]$. Accuracy for guess responses was $76 \%\left[t_{(17)}=4.8, P=\right.$ 0.0002 vs. chance] when guessing was encouraged and $46 \%$ $\left[t_{(17)}=0.5, P=0.61\right.$ vs. chance $]$ when confident responses were encouraged, and guesses were significantly more accurate in the former condition than in the latter $\left[t_{(17)}=2.8, P=0.01\right]$. Accuracy findings were essentially the same as when all subjects were considered, indicating that effects were not driven by low trial counts.

Encouraging guessing versus confident responding also influenced the prevalence of metamemory responses that subjects made using standard "remember/know" procedures (see the Materials and Methods). Metamemory response rates and accuracy results are provided in Table 1 . Considering all subjects, "remember" responses, which were used to indicate the experience of recollection, were registered for $27 \%$ of the trials when guessing was encouraged, and for $36 \%$ of the trials when confident responding was encouraged $\left[t_{(23)}=4.1, P=0.0006\right]$. Remember response accuracy was high regardless of what kind of responding was encouraged [76\% when guessing was encouraged, and $80 \%$ when confident responding was encouraged; $\left.t_{(23)}=0.4, P=0.70\right]$. "Know" responses, which were used to indicate the experience of familiarity, were registered at similar rates when guessing was encouraged versus when confident responding was encouraged [ 48 vs. $52 \%$, respectively, $t_{(23)}=1.4, P=$ $0.18]$. Likewise, the accuracy of know responses did not differ based on instructional condition $\left[t_{(23)}=0.9, P=0.35\right]$.

In our previous experiment in which remember, know, and guess responses were collected on each trial (Voss and Paller 2009), we found that remember and guess responses were made with approximately equivalent accuracy, and both remember and guess responses were more accurate than know responses. In the current experiment, guess responses were significantly more accurate than know responses, but only when guessing was encouraged $\left[t_{(23)}=2.9, P=0.008\right]$. When confident responding was encouraged, guess responses were significantly less accurate than know responses $\left[t_{(23)}=2.2, P=0.04\right]$. The accuracy of remember responses versus guess responses did not differ significantly when guessing was encouraged $\left[t_{(23)}=0.1, P=0.91\right]$, but remember responses were significantly more accurate than guess responses when confident responding was encouraged $\left[t_{(23)}=\right.$ $4.7, P=0.0001]$. Thus, the pattern of accuracy for remember, know, and guess responses reported in Voss and Paller (2009) was replicated only when guessing was encouraged (Fig. 3).

The pattern of accuracy for remember, know, and guess responses summarized in Figure 3 also provides evidence against the possibility that encouraging guessing led to a relative boost in guessing accuracy for the trivial reason that this encouragement led to the use of different criteria for these introspective 


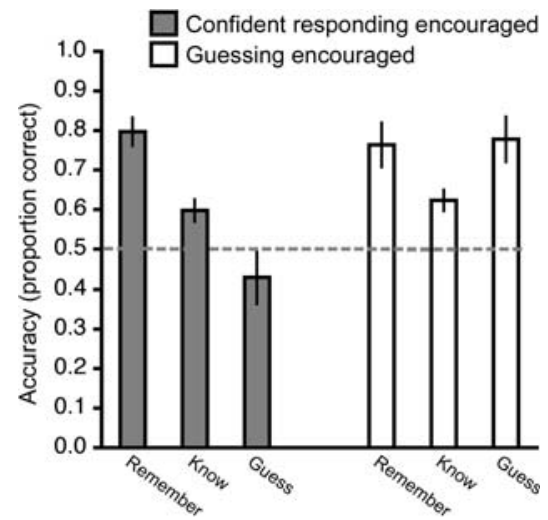

Figure 3. Recognition accuracy in the two encouragement conditions. Mean accuracy is shown for remember, know, and guess responses when confident responding was encouraged versus when guessing was encouraged. The accuracy expected by chance was 50\%. Error bars indicate \pm SE.

response categories. That is, encouraging guessing compared with encouraging confidence might have led to a more liberal criterion for registering guess responses, such that some trials that would have garnered "know" responses when confidence was encouraged were instead registered as guesses. Figure 3 shows that, when confidence was encouraged, accuracy was highest for remember responses, less accurate for know responses, and least accurate for guess responses, but, when guessing was encouraged, accuracy showed a "U-shaped" pattern, with remember responses and guesses associated with higher accuracy than know responses. Furthermore, as indicated above, remember and know responses did not differ in accuracy for the two encouragement conditions. It is therefore not plausible for the increase in guessing accuracy when guessing was encouraged to have been the result of simply a greater tendency to use the guess response instead of the know response.

Subjects were instructed to withhold recognition responding until after the 2-sec stimulus presentation, as in previous experiments (Voss et al. 2008; Voss and Paller 2009; Jeneson et al. 2010). The mean response times following this 2 -sec interval for remember, know, and guess responses when guessing was encouraged were $502 \mathrm{msec}(\mathrm{SE}=121), 264 \mathrm{msec}(\mathrm{SE}=152)$, and $433 \mathrm{msec}$ $(\mathrm{SE}=95)$, respectively. When confident responding was encouraged, these values were: $375 \mathrm{msec}(\mathrm{SE}=87), 443 \mathrm{msec}(\mathrm{SE}=151)$, and $386 \mathrm{msec}(\mathrm{SE}=162)$, respectively. No pairwise comparisons between any two conditions reached significance (all $P$ values $>0.09 ; P$-value range $0.09-0.94)$.

\section{Discussion}

Our results supported the veracity of implicit recognition. When guessing was stressed, guessing accuracy exceeded the accuracy of responding based on subjective familiarity, and reached the level of recollection-based recognition. When confidence was stressed, accurate guessing was eliminated.

Encouraging subjects to guess was associated with substantially more guess responses compared with encouraging subjects to respond confidently. In contrast, remember responses were more prevalent when confident responding was encouraged compared with when guessing was encouraged. The prevalence of know responses was not influenced by the encouragement manipulation. The accuracy of guesses (but not of remember or know responses) was increased by encouraging guessing versus confident responding. Guess accuracy reached the remarkably high level of $77.7 \%$ correct when guessing was encouraged, but was no better than chance (50\%) when confident responding was encouraged.

These findings support our contention that the way in which subjects are encouraged to approach the recognition memory test can substantially influence not only how often they guess, but also the accuracy of guess responses. Figure 4 shows the accuracy for guess responses averaged across all individual experiments and conditions for our original reports (Voss et al. 2008; Voss and Paller 2009), for the report by Jeneson et al. (2010), and for the two encouragement conditions in the current experiment. When guessing was encouraged in the current experiment, guess accuracy was on par with guess accuracy in our previous reports. When confident responding was encouraged, guess accuracy was at chance, as it was in the experiments reported by Jeneson et al. (2010). Even though Jeneson and colleagues took great care in replicating our design, including use of the same stimuli and some of the verbal instructions, we consider it likely that the manner in which the tests were administered differed between our original experiments and those conducted by Jeneson and colleagues. When we provided them with our written instructions, we did not realize the potential importance of the emphasis on and periodic reminders about guessing. Fortunately, this discrepancy led directly to the present experiment, which now clarifies the importance of aspects of our procedure that we did not appreciate earlier. We suggest that guessing was encouraged through regular experimenter-subject interactions in our prior experiments, and that this is essential for obtaining implicit recognition.

This insight might be taken to imply that implicit recognition is indeed an elusive phenomenon, in that it may be sensitive to subtle variations in the manner in which instructions are delivered. However, it is important to consider that instructions can be comprehended in ways that radically change the manner in which a task is approached. The comprehension of instructions varies according to not only which portions are emphasized by the experimenter, but potentially also other ways in which

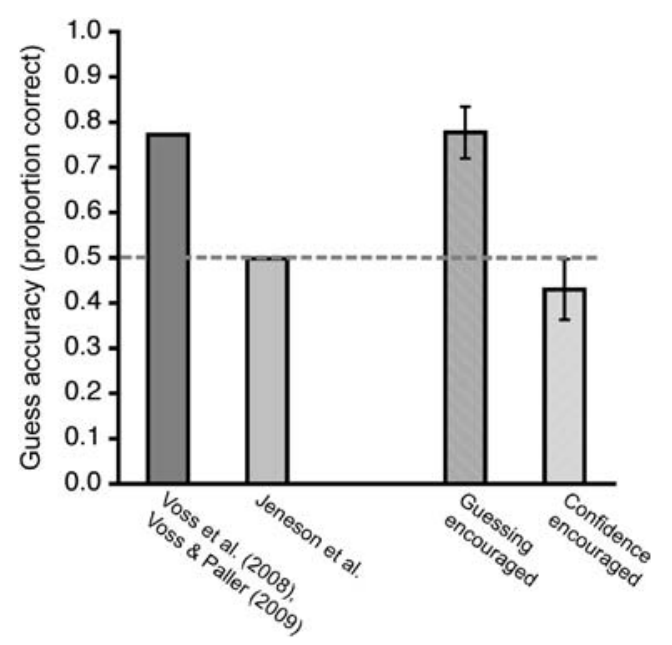

Figure 4. The relationship between the extent to which guessing is encouraged in an experiment and whether the experiment reveals implicit recognition. Accuracy of guess responses are shown averaged across all experimental conditions and across all individual experiments reported in (1) Voss et al. (2008) and Voss and Paller (2009), and (2) the experiments by Jeneson et al. (2010). Also shown is the mean accuracy of guesses in the current experiment when guessing was encouraged versus when confident responding was encouraged. The accuracy expected by chance was $50 \%$ in all experiments. Error bars indicate \pm SE. 
experimenters interact with subjects (e.g., the rapport they establish, the extent to which they interact with subjects to make them comfortable with the procedures, the extent to which they motivate subjects to perform the task, and so on). Although experimental designs are commonly intended to mitigate these factors, it is conceivable that they nonetheless influence performance in some circumstances, such as when "guessing" can have two easy-to-adopt yet diametrically opposed meanings. We conclude, based on the current results, that instructions encouraging guessing can produce responses based on rapid-onset signals of visual fluency, without competing influences from explicit retrieval processes dependent on deliberate retrieval effort. In contrast, when confident responding is emphasized, subjects may attempt to deliberately retrieve relevant episodic information, and then register a guess response only when this fails. These guesses would thus tend to be made on a random basis following the failure of deliberate retrieval, rather than on the basis of visual fluency. This proposal is consistent with evidence that accuracy can be enhanced by encouraging subjects to adopt lax response criteria, even in patients with global amnesia (Verfaellie et al. 2001). Other studies have also documented results of encouraging nonanalytic, guess-like strategies (Whittlesea and Price 2001; Willems et al. 2008; Willems and Van der Linden 2009).

By this scenario, response times could conceivably be used to distinguish between guessing based on failed episodic retrieval (ordinary guessing) and guessing based on visual fluency signals (guessing informed by implicit knowledge). ${ }^{5}$ Unfortunately, response times were unhelpful for making this assessment in all of these experiments because the procedures required responses to be withheld for $2 \mathrm{sec}$ after stimulus onset. On the other hand, electroencephalographic data described below are consistent with the notion that processing relevant for implicit recognition occurs earlier than processing relevant for explicit recognition.

Even taking the above considerations into account, it should still be acknowledged that implicit recognition is a relatively exacting phenomenon to demonstrate. Whereas many memory phenomena are highly robust and easy to replicate (e.g., effects of elaborative semantic encoding on subsequent recall), other memory phenomena depend on multiple design features, some of which may be highly atypical or not well characterized. Decades of research support the commonsense notion that accuracy and confidence are highly correlated in most situations. Accordingly, implicit recognition is a phenomenon that is operative in only special circumstances-it may be sensitive to subtle variations in experimental parameters that we have only begun to identify. We nonetheless contend that implicit recognition constitutes an important phenomenon at the border of implicit and explicit memory, and that investigations of this phenomenon could provide valuable insights into the processes that support recognition memory.

To this end, we describe a strategy that we feel is a promising way to pursue implicit recognition and the contributions it may make to recognition memory at large. The general goal is to characterize the mechanisms hypothesized to contribute to

\footnotetext{
${ }^{5}$ Jeneson et al. (2010) used another tactic to look for implicit recognition by examining recognition accuracy in subjects exhibiting the highest prevalence of guessing. But when they considered data only from subjects with a relative propensity to guess in their Experiment 2, there was no evidence for accurate guessing. To speculate: The shortcoming of this tactic might be that guessing in these subjects, as in other subjects in the group, resulted from the failure of deliberate retrieval, and so was not based on implicit knowledge. These subjects might have guessed more often than others because they were the least competent at storing and/or retrieving the relevant information, such that they experienced episodic retrieval failure more often.
}

implicit recognition, which can then be used to assess the extent to which these processes contribute to recognition overall. Because evidence for implicit recognition was obtained only on forced-choice recognition tests, and only when targets and corresponding foils were highly perceptually similar (Voss et al. 2008), we hypothesized that implicit recognition is distinct from retrieval of episodic information and heavily dependent on the repetition-related fluency of perceptual processing, as the relative visual fluency of the target compared with the foil could be used to make an accurate recognition response only in these circumstances.

Electrophysiological correlates of highly accurate guess responses supported this perceptual fluency hypothesis of implicit recognition (Voss and Paller 2009). Highly accurate guesses were associated with repetition effects on event-related potentials (ERPs) that were rapid in onset $(\sim 100-300 \mathrm{msec}$ after stimulus onset), negative in sign (less positive for repeat targets relative to novel foils), and maximal at electrodes situated above the occipital cortex. These features all match putative ERP correlates of perceptual fluency obtained in different circumstances, in which special procedures were used to isolate perceptual implicit memory for faces (Paller et al. 2003). In striking contrast, ERP correlates of explicit memory (when subjects reported either recollection or familiarity) included later-onset positive shifts in ERPs at parietal electrodes (Voss and Paller 2009), consistent with the known ERP correlates of explicit memory obtained in a wide variety of circumstances (Voss and Paller 2008). Thus, ERP correlates of accurate recognition responses dissociated according to whether subjects reported guessing versus responding with confidence and awareness of retrieval (Fig. 5); ERP correlates of accurate guesses were consistent with the putative role of perceptual fluency in implicit recognition.

To further explore the possibility that implicit knowledge in the form of visual fluency was responsible for accurate recognition guesses, we obtained ERP correlates of perceptual priming for the same kaleidoscope stimuli that were used in our studies of implicit recognition (Voss and Paller 2010). Kaleidoscope images appeared in an implicit memory test that required perceptual judgments to each stimulus-subjects rated the number of colors comprising each stimulus, which varied from three to five. Perceptual priming was evident as significantly faster and more accurate discrimination responses to repeat images versus novel images. Crucially, ERP correlates of perceptual priming closely resembled those of accurate guesses during recognition testing, and, in both cases, ERPs were distinct from those of explicit memory (Fig. 5). These rapid-onset negative ERP effects can therefore be interpreted as neural correlates of visual fluency, which can drive facilitated perceptual responses during a priming test, and can also lead to accurate guesses in forced-choice recognition tests with perceptually similar target/foil pairs.

It should be noted that other studies have already investigated influences of perceptual fluency on recognition memory. Enhancing perceptual fluency leads to a bias to indicate that an item has been seen previously (i.e., endorse an item as "old"), irrespective of whether the item was actually seen previously (i.e., for both repeat and novel items, as long as target and foil stimuli have the same relevant features) (e.g., Whittlesea and Williams 1998; Verfaellie and Cermak 1999; Kleider and Goldinger 2004). That is, perceptual fluency biases "old" endorsements such that they are more prevalent for both old and new items, and therefore does not generally increase the accuracy of recognition responses. A notable exception is when recognition tests are constructed such that this bias to respond "old" due to perceptual fluency actually leads to the correct response in the recognition test (e.g., Keane et al. 2006). Interestingly, one previous study found that the bias of perceptual fluency on recognition responses was 

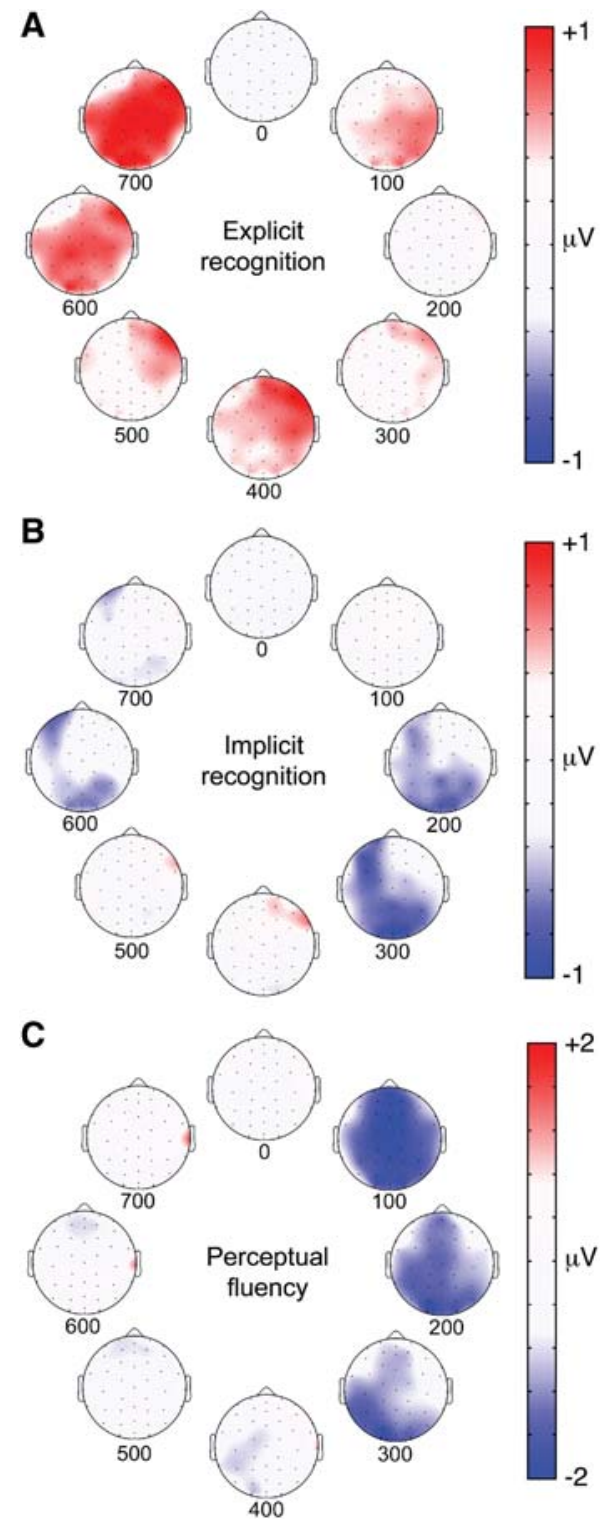

Figure 5. Neural correlates of explicit recognition, implicit recognition, and implicit perceptual fluency. ERP old/new effects are shown for recognition responses based on an explicit feeling of familiarity $(A)$ versus highly accurate guesses $(B)$. (C) The latter ERP effects are strikingly similar to those related to perceptual fluency obtained in an implicit perceptual priming test. Topographic plots of the old/new differences are shown for each of these three memory types averaged for successive 100-msec intervals starting at stimulus onset. ERP difference values are indicated by coloration. Data are from Voss and Paller (2009, 2010). Figure adapted from Voss and Paller (2010) and reprinted with express permission from the authors.

most prevalent for guess responses (Tunney and Fernie 2007), consistent with our findings of accurate guess responses based on perceptual fluency. A notable difference between our studies and those showing "old" biases due to perceptual fluency is that our tests were constructed specifically so that the fluency bias could be used to select the correct response, as in the study by Keane et al. (2006), and more generally in experiments showing accurate forced-choice perceptual discriminations based on guesses (Adams 1957).
Conroy et al. (2005) presented evidence that signals of perceptual fluency provide little benefit to recognition. However, this limitation might be more relevant for the standard recognition circumstances they described, but less so as circumstances become less standard. It is thus conceivable that implicit recognition can contribute to performance in various other circumstances but be difficult to identify because the benefit does not constitute the major influence on performance. When circumstances are highly unusual (forced-choice format and perceptually similar target/foil pairs) implicit contributions can become frequent enough to reach the very high level needed to clearly impact performance so as to identify implicit recognition, and potentially also play a role in confidence/accuracy inversions and perceptual discrimination without awareness.

We therefore concur with Jeneson et al. (2010) in advocating that future studies focus on circumstances in which perceptual fluency influences recognition, with special emphasis on responding in the absence of retrieval awareness. Importantly, there may be more than one way to guess; some guesses simply signify the complete absence of any retrieved information, whereas other guesses may be based on information that is not available to be subjectively experienced, as in the replicable influences of perceptual fluency discussed in this study. Thus, future studies investigating guess responses should incorporate the methodological information presented here, given that encouraging guessing is a crucial factor in implicit recognition. We also advocate experimental approaches that begin to probe the neural mechanisms of fluency by identifying neural correlates of the influence of fluency on recognition and neural correlates of fluency during an implicit memory test, as in our studies using electrophysiological measures (Voss and Paller 2009, 2010). By focusing on how perceptual fluency influences recognition, and the special conditions in which fluency can lead to accurate responding, future studies on implicit recognition and its neural substrates can inform models of the interrelationships among implicit memory, recognition, and retrieval awareness.

\section{Materials and Methods}

\section{Subjects}

Behavioral data were collected from 24 Northwestern University students, ages 18-27 yr, 19 female, who were paid for their participation.

\section{Stimuli}

Visual stimuli included kaleidoscope images created using previously described methods (Voss et al. 2008). Kaleidoscope images were each comprised of three distinct colors, and were organized into 60 pairs. The two members of each pair were highly similar. One member of each pair was assigned as the repeat target and the other as the novel foil, with assignments counterbalanced across subjects. An additional 24 items were used as buffers. Stimuli were further grouped into six sets (10 pairs plus four buffers). The same three colors were used for each member of a set, such that color per se was not a reliable cue for recognition. One color set was used in each study/test block.

\section{Procedure}

The experiment comprised six study/test blocks, during which subjects studied 14 kaleidoscope images and then were tested for the middle 10 images (the first two and last two images were not tested to account for primacy and recency effects). During the study phase, each image was presented for $2000 \mathrm{msec}$ with a 1500 -msec ISI. A randomly selected spoken numeric digit onset simultaneously with each kaleidoscope image. Subjects performed a divided-attention task using these digits. On each trial, 
subjects responded with a two-choice button response according to whether the number presented on the previous trial was odd or even (one-back divided-attention task). Subjects practiced this task prior to the experimental blocks until accuracy was $100 \%$ for 20 consecutive trials.

Two-alternative forced-choice recognition tests were administered after a delay of $\sim 30$ sec during which verbal instructions were provided. Targets and foils were presented simultaneously on the screen for $2 \mathrm{sec}$, one on the left and one on the right, with $\sim 5^{\circ}$ of visual angle separating them. The location of the target was assigned at random for each target/foil pair, with an equivalent number of targets on the right as on the left in each block. Stimuli disappeared after the 2 -sec viewing interval, at which time subjects were to immediately press one of two buttons to indicate the location of the target. After registering this recognition response, subjects indicated whether the recognition decision was based on a feeling of recollection (remember response), familiarity (know response), or pure guessing without any confidence or awareness of remembering (guess response). Instructions closely mirrored those used in our previous studies.

Guessing was encouraged for half of the study/test blocks, and confident responding was encouraged for half of the study/ test blocks. Guessing was encouraged for either the first three blocks or the last three blocks, counterbalanced across subjects. The instructions and interactions with the subjects used to encourage guessing were highly similar to the general procedures in our previous reports, although tests were administered by two individuals who were not involved with the previous data collection.

In advance of blocks in which guessing was encouraged, the experimenter informed subjects that the test would be very difficult, and encouraged them to try to respond as accurately as possible, emphasizing that guessing was acceptable. The experimenter repeatedly reassured the subjects of the appropriateness of guess responses during every break between study and test sessions (i.e., "just do your best and guess if you need to"), while also reminding them of the general instructions. In contrast, confident responding was encouraged by asking subjects to try to respond as accurately as possible and to guess only as a last resort (i.e., "it is very important that you try to respond as accurately as possible and to guess only when absolutely necessary"). Subjects were also instructed that, if a guess did occur, it was important to register it as such, but confident responding over guessing was encouraged during every break between study and test sessions. The complete script that was read to subjects during the experiment is available from the authors by request.

\section{Acknowledgments}

Financial support was provided by a Beckman Institute Postdoctoral Fellowship award to J.L.V., and by a grant from NSF (BCS-0818912). Many thanks to Susan Florczak and Jessica Creery for collecting data.

\section{References}

Adams JK. 1957. Laboratory studies of behavior without awareness. Psychol Bull 54: 383-405.

Conroy MA, Hopkins RO, Squire LR. 2005. On the contribution of perceptual fluency and priming to recognition memory. Cogn Affect Behav Neurosci 5: 14-20.

Craik FIM, Lockhart RS. 1972. Levels of processing: A framework for memory research. J Verb Learn Verb Behav 11: 671-684.

Dijksterhuis A, Bos MW, Nordgren LF, van Baaren RB. 2006. On making the right choice: The deliberation-without-attention effect. Science 311: $1005-1007$.
Dijksterhuis A, Bos MW, van der Leij A, van Baaren RB. 2009. Predicting soccer matches after unconscious and conscious thought as a function of expertise. Psychol Sci 20: $1381-1387$.

Dobbins IG, Kroll NEA, Liu Q. 1998. Confidence-accuracy inversions in scene recognition: A remember-know analysis. J Exp Psychol Learn Mem Cogn 24: 1306-1315.

Fullerton GS, Cattell JM. 1892. On the perception of small differences. University of Pennsylvania Press, Philadelphia, PA.

Hassin RR, Uleman JS, Bargh JA, ed. 2005. The new unconscious. Oxford University Press, New York.

Heathcote A, Freeman E, Etherington J, Tonkin J, Bora B. 2009. A dissociation between similarity effects in episodic face recognition. Psychonomic Bull Rev 16: 824-831.

Heathcote A, Bora B, Freeman E. 2010. Modeling choice-similarity effects in episodic recognition. J Mem Lang 62: 183-203.

Jeneson A, Kirwan CB, Squire LR. 2010. Recognition without awareness: An elusive phenomenon. Learn Mem (this issue). doi: 10.1101/ $\operatorname{lm} .1815010$.

Keane MM, Orlando F, Verfaellie M. 2006. Increasing the salience of fluency cues reduces the recognition memory impairment in amnesia. Neuropsychologia 44: 834-839.

Kelley R, Wixted J. 2001. On the nature of associative information in recognition memory. J Exp Psychol Learn Mem Cogn 27: $701-722$.

Kleider HM, Goldinger SD. 2004. Illusions of face memory: Clarity breeds familiarity. J Mem Lang 50: 196-211.

Mulligan NW. 1998. The role of attention during encoding in implicit and explicit memory. J Exp Psychol Learn Mem Cogn 24: 27-47.

Paller KA, Huston CA, Miller BB, Boehm SG. 2003. Neural manifestations of memory with and without awareness. Neuron 38: 507-516.

Peirce CS, Jastrow J. 1884. On small differences of sensation. Mem Nat Acad Sci 3: 73-83.

Schooler JW, Ohlsson S, Brooks K. 1993. Thoughts beyond words: When language overshadows insight. J Exp Psychol Gen 122: 166-183.

Sidis B. 1898. The psychology of suggestion. Appleton, New York.

Stroh M, Shaw AM, Washburn MF. 1908. A study in guessing. Am J Psychol 19: $243-245$.

Tulving E. 1981. Similarity relations in recognition. J Verb Learn Verb Behav 20: $479-496$.

Tunney RJ, Fernie G. 2007. Repetition priming affects guessing not familiarity. Behav Brain Func 3: 40-46.

Verfaellie M, Cermak LS. 1999. Perceptual fluency as a cue for recognition judgments in amnesia. Neuropsychology 13: 198-205.

Verfaellie M, Giovanello KS, Keane MM. 2001. Recognition memory in amnesia: Effects of relaxing response criteria. Cogn Affect Behav Neurosci 1: $3-9$.

Voss JL, Paller KA. 2008. Brain substrates of implicit and explicit memory: The importance of concurrently acquired neural signals of both memory types. Neuropsychologia 46: 3021-3029.

Voss JL, Paller KA. 2009. An electrophysiological signature of unconscious recognition memory. Nat Neurosci 12: 349-355.

Voss JL, Paller KA. 2010. Real-time neural signals of perceptual priming with unfamiliar geometric shapes. J Neurosci 30: 9181-9188.

Voss JL, Baym CL, Paller KA. 2008. Accurate forced-choice recognition without awareness of memory retrieval. Learn Mem 15: 454-459.

Weiskranz L. 1990. Blindsight: A case study and implications. Oxford University Press, New York.

Whittlesea BW, Price JR. 2001. Implicit/explicit memory versus analytic/ nonanalytic processing: Rethinking the mere exposure effect. Mem Cognit 29: $234-246$.

Whittlesea BW, Williams LD. 1998. Why do strangers feel familiar, but friends don't? A discrepancy-attribution account of feelings of familiarity. Acta Psychologia (Amst) 98: 141-165.

Willems S, Van der Linden M. 2009. Experimental dissociations between memory measures: Influence of retrieval strategies. Conscious Cogn 18: $39-55$.

Willems S, Salmon E, Van der Linden M. 2008. Implicit/explicit memory dissociation in Alzheimer's disease: The consequence of inappropriate processing? Neuropsychology 22: 710-717.

Yonelinas AP. 2001. Consciousness, control, and confidence: The 3 Cs of recognition memory. J Exp Psychol Gen 130: 361-379.

Received June 4, 2010; accepted in revised form July 21, 2010. 


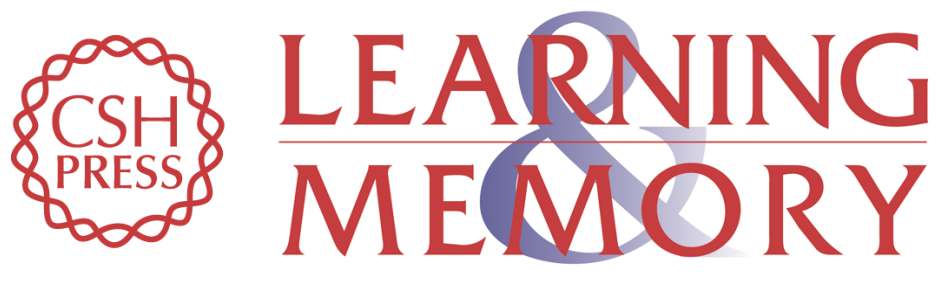

\section{What makes recognition without awareness appear to be elusive? Strategic factors that influence the accuracy of guesses}

Joel L. Voss and Ken A. Paller

Learn. Mem. 2010, 17:

Access the most recent version at doi:10.1101//m.1896010

References This article cites 29 articles, 3 of which can be accessed free at:

http://learnmem.cshlp.org/content/17/9/460.full.html\#ref-list-1

License

Email Alerting Receive free email alerts when new articles cite this article - sign up in the box at the Service top right corner of the article or click here. 